\title{
Geodesy and geomatics: the cutting edge
}

\author{
Fernando Sansò $\cdot$ Mattia Crespi
}

Received: 23 April 2015/Accepted: 23 April 2015/Published online: 16 May 2015

(C) Accademia Nazionale dei Lincei 2015

Geodesy is a very old science, born in Greece, with the Pythagorean hypothesis of the spherical shape of the Earth, and developed in Egypt, with the famous and unbelievable accurate determination (around $10 \%$ ) of the radius of the Earth in the third century B.C. by Eratosthenes of Cyrene, a mathematician chief of the Library of Alexandria. Actually this was the classical form of "Higher Geodesy", studying at large the shape of the Earth, but "Applied Geodesy", meant as surveying at a local level, was even much older, having been practised by ancient societies like Sumerian and Egyptians, then continuously further by Greeks and Romans.

Since the ancient era, only the remarkable work by the Persian mathematician and astronomer Al-Biruni lived in middle age around 1000 A.C. is worth to be mentioned, as he re-determined the radius of the Earth with an accuracy better than $1 \%$, following an approach different from Eratosthenes.

Later geodesy greatly benefited of the scientific revolution and developed at the beginning of the modern era, together with astronomy, mechanics and modern

Peer reviewed version of the paper presented at conference on Geodesy and Geomatics held at Accademia Nazionale dei Lincei in Rome on June 3, 2014.

F. Sansò $(\bowtie)$

Dipartimento di Ingegneria Civile e Ambientale, Politecnico di Milano, P.zza Leonardo da Vinci, 32, 20133 Milan, Italy

e-mail: fernando.sanso@polimi.it

M. Crespi

Area di Geodesia e Geomatica, Dipartimento di Ingegneria

Civile, Edile e Ambientale, Università di Roma "La Sapienza",

Via Eudossiana, 18, 00184 Rome, Italy

e-mail: mattia.crespi@uniroma1.it calculus; be it enough to mention the famous dispute on the ellipsoidal shape of the Earth between Newton (oblate) and Cassini (oblong), solved in favour of Newton thanks to the French Geodetic Missions to Lapland and Ecuador; in that occasion, Bouguer also realized that mountains impact the gravity direction.

Basic instruments of geodesy have been for long (through clearly with a precision increasing in time) the gravimeter and the plumb line direction for gravity measurements, the theodolite (a pair of goniometers with a telescope of reduced dimensions) for angle measurements, the rods and wires for distance measurements. It is worth to be mentioned that, for long time, geodetic measurements were the most accurate ones in science and technology, what boosted remarkably "the art" of measurements modelling and the theoretical investigations about the best ways to cope with observations unavoidable errors.

Basic scientific tools have been Newton's law, geometrical optics, Euclidean and ellipsoidal geometry and specially the theory of least squares, dating back to the times of Legendre and Gauss, and capable of accommodating the inconsistencies between theoretical models and measurements.

This theory in particular, although used in other disciplines, has been the flag of geodesy as it is in its framework that it developed to a full generality, with unequalled numerical applications.

From the 60s of the past century, however, like many other disciplines, geodesy has started an unprecedented period of accelerated development, where the dialectics between new technological achievements and new mathematical theories has produced a spectacular enhancement and a considerable widening of the areas of applications.

Here we try to list what appear to be the main factors, though with no pretence to be complete: 


\begin{tabular}{|c|c|}
\hline Technology and measurements & $\begin{array}{l}\text { Theory enhancement (within and } \\
\text { around geodesy) }\end{array}$ \\
\hline \multicolumn{2}{|l|}{ Electronic hardware } \\
\hline $\begin{array}{l}\text { Laser, clocks, signal correlators, } \\
\text { sensor arrays, radar, } \\
\text { accelerometers and gyros }\end{array}$ & $\begin{array}{l}\text { 3D geodesy and global } \\
\text { positioning } \\
\text { Deformation monitoring theory } \\
\text { Large number of integers } \\
\text { estimation (GNSS, SAR) } \\
\text { Navigation (aerial, marine, } \\
\text { terrestrial) }\end{array}$ \\
\hline \multicolumn{2}{|l|}{ Computers } \\
\hline $\begin{array}{l}\text { Computational speed, storing } \\
\text { capacity, programming } \\
\text { languages, software }\end{array}$ & $\begin{array}{l}\text { Field approximation theory } \\
\text { (functional analysis) } \\
\text { Random fields and their } \\
\text { prediction } \\
\text { Large system of equations } \\
\text { solution } \\
\text { Image analysis, 3D modelling } \\
\text { and Remote Sensing } \\
\text { Databases theory and GIS }\end{array}$ \\
\hline \multicolumn{2}{|l|}{ Space technology } \\
\hline $\begin{array}{l}\text { Launchers attitude and speed } \\
\text { control, miniaturization }\end{array}$ & $\begin{array}{l}\text { Celestial mechanics for low } \\
\text { orbiting satellites navigation } \\
\text { (aerial, marine, terrestrial) } \\
\text { 4D and real time Earth } \\
\text { information theory }\end{array}$ \\
\hline
\end{tabular}

Not only this short list is incomplete, but, although its typographical layout suggests that the driving factor is technology and, on the right, there are the corresponding areas of development of theory within and around geodesy, reality is certainly more complex.

In fact, items at different levels are in any way interwoven by more or less strong interactions. Yet, the list is useful in giving the idea of the large jump over the last 50 years.

The field has been growing and growing to such an extent that a longer designation was deemed urgent, so much so that nowadays the characterization "Geodesy and Geomatics" is generally accepted. In this context "Geodesy" represents the methodological core, whereas "Geomatics" wants to underline the continuously widening applications together with the contribution of electronics and informatics to the development of this science.

However, all this matter would simply be an agglomerate of notions and techniques, namely not a Science, if there would not be a common line of principles and tools, guiding such an evolution, and a common object of interest of our investigations.

The common object we can say is the Earth in the sense of geometry, from the surface (or little below) to the closer space at all scales from 1 to $10^{8} \mathrm{~m}$, embedded in its gravity field which is, so to say, part of its figure.
The tools are all the mathematical (geometrical, functional, probabilistic, numerical, etc.) instruments and the physical (relativistic, mechanical, electromagnetical, etc.) laws that constitute an arsenal where geodesy and geomatics performs a selection of, or develops by its own, appropriate tools, driven by the needs of describing and using information provided by technological evolution.

Geodesy and geomatics is so to say an adaptive discipline where the scientific model is expanding according to the need of explaining and using new measurements; the characteristic of this science though is that the boundary between known and unknown geodetic universe is in reality a vast area dominated by uncertainty, which is the object of intensive research to obtain the best probabilistic modelling and the most accurate results in terms of positioning of points, gravity field determination, surface reconstruction, image interpretation, etc. in space (3D) and time $(+1 \mathrm{D})$.

Summarizing the above description in one image only one could use the $3 \mathrm{M}$ commutative diagram below:

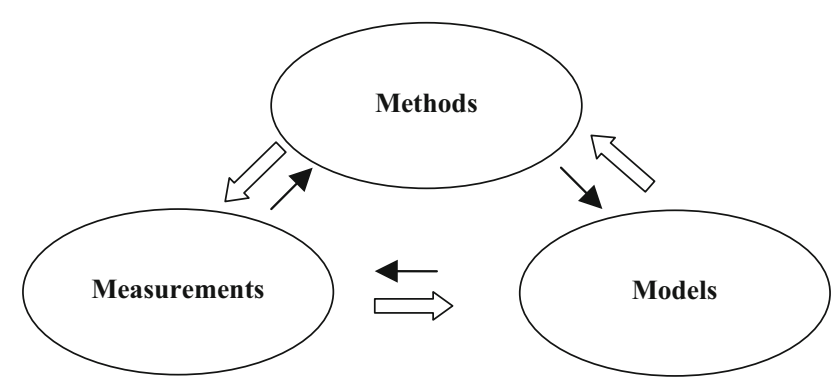

This is what geodesy and geomatics do with the main counterclockwise flow, interacting with other sciences, primarily geophysics but also astronomy, physics and mathematics with its main branches from functional analysis through probability theory to numerics. A slow of science development always adapting models so as to account for the main components, but in a way sufficiently simple that measurements become treatable by our methods.

In front of the fantastic results achieved and of the many new questions that they pose, we have decided to organize a 1-day workshop of which this volume constitutes the Proceedings, with a twofold purpose: on the one hand to help the younger Italian generation of researchers on our subject, to get in contact with the new challenges of geodesy and geomatics; on the other hand to bring to the Accademia dei Lincei, as the maximum temple of scientific (and humanistic) culture in Italy, a synthetic picture of the achievements of our field in the last decades.

We would like to thank Prof. Michele Caputo, the head of the Scientific Committee of the workshop, for his 
illuminated guidance. We are deeply grateful to Prof. Lamberto Maffei, the President of the Accademia, and its governing board for having hosted this event.

We would also like to thank the Politecnico di Milano and the University of Roma "La Sapienza" for the economical support.
Last, but not least, let us express our great satisfaction for having gathered on June 3, 2014, about 120 people, the large majority of researchers in geodesy and geomatics in Italy; our sincere thanks to them. 